\title{
Array Tomography: High-Resolution Three-Dimensional Immunofluorescence
}

\author{
Kristina D. Micheva, Nancy O'Rourke, Brad Busse, and Stephen J. Smith
}

\section{INTRODUCTION}

Array tomography, which is described in this article, is a volumetric microscopy method based on physical serial sectioning. Ultrathin sections of a plastic-embedded tissue are cut using an ultramicrotome, bonded in an ordered array to a glass coverslip, stained as desired, and imaged. The resulting two-dimensional image tiles can then be reconstructed computationally into three-dimensional volume images for visualization and quantitative analysis. The minimal thickness of individual sections permits high-quality rapid staining and imaging, whereas the array format allows reliable and convenient section handling, staining, and automated imaging. Also, the physical stability of the arrays permits images to be acquired and registered from repeated cycles of staining, imaging, and stain elution, as well as from imaging using multiple modalities (e.g., fluorescence and electron microscopy). Although the fabrication procedures can be relatively difficult, the high resolution, depth invariance, and molecular discrimination offered by array tomography justify the effort involved. Array tomography makes it possible to visualize and quantify previously inaccessible features of tissue structure and molecular architecture.

\section{RELATED INFORMATION}

Procedures for processing, sectioning, and staining tissue are described in Array Tomography: Rodent Brain Fixation and Embedding (Micheva et al. 2010a), Array Tomography: Production of Arrays (Micheva et al. 2010b), and Array Tomography: Immunostaining and Antibody Elution (Micheva et al. 2010c). The techniques for image acquisition and processing three-dimensional reconstruction are available in Array Tomography: Imaging Stained Arrays (Micheva et al. 2010d) and Array Tomography: Semiautomated Image Alignment (Micheva et al. 2010e).

\section{BACKGROUND AND SIGNIFICANCE}

Our understanding of tissue function is constrained by incomplete knowledge of tissue structure and molecular architecture. Genetics, physiology, and cell biology make it overwhelmingly clear that all cell and tissue function depends critically on the composition and precise three-dimensional configuration of subcellular organelles and supramolecular complexes, and that such structures can consist of very large numbers of distinct molecular species. Unfortunately, the intricacies of tissue molecular architecture badly outstrip the analytical capability of all presently known tissue imaging methods.

Array tomography is a new high-resolution three-dimensional microscopy method based on constructing and imaging two-dimensional arrays of ultrathin (70-200 nm thickness) specimen sections on solid substrates. "Tomography" derives from the Greek words tomos (to cut or section) and graphein (to write); the term "array tomography" thus simply connotes the "writing" of a volume image from an array of slices. Array tomography, developed with neuroscience applications in mind (e.g., Smith 2007; Stephens et al. 2007; Koffie et al. 2009), allows immunofluorescence imaging of tissue samples with resolution, quantitative reliability, and antibody multiplexing capacity that is greatly superior to previous tissue immunofluorescence methods (Micheva and Smith 2007).

Adapted from Imaging: A Laboratory Manual (ed. Yuste). CSHL Press, Cold Spring Harbor, NY, USA, 2010.

Cite as: Cold Spring Harb Protoc; 2010; doi:10.1101/pdb.top89

www.cshprotocols.org 


\section{ARRAY TOMOGRAPHY PROCEDURES}

The array tomography method (Fig. 1) begins with the chemical fixation of the specimen (Step 1), followed by dissection and embedding in resin (e.g., LR White; Step 2). Specimen blocks are then mounted in an ultramicrotome chuck, trimmed, and prepared for ultrathin sectioning (Step 3). Block preparation includes careful trimming of the block edges and application of a tacky adhesive to the top and bottom block edges. This adhesive spontaneously forms a stable splice between successive serial sections as they are cut by the microtome's diamond blade. The automated cycling of a standard ultramicrotome produces a ribbon up to $45 \mathrm{~mm}$ in length, which can consist of more than 100 serial sections held on a water surface. Ribbons are then transferred manually to the surface of a specially coated glass coverslip (Step 4). The resulting array can be stained using antibodies or other desired reagents (Step 5) and imaged using fluorescence microscopy (Step 6). The minimal thickness of array sections promotes rapid and excellent staining and imaging, whereas the array format allows a large number of serial sections to be handled conveniently and reliably. Individual two-dimensional section images are then stitched and aligned by computer into volumetric image stacks (Step 7), permitting three-dimensional image visualization and analysis (see, e.g., Fig. 2); these stacks can be archived electronically for subsequent analysis (Step 8). Compared with many other imaging methods, array tomography procedures presently are relatively complex and demanding. However, each step lends itself to automated and highly parallel implementations, and for many applications, the advantages justify the extra effort.

\section{Resolution}

The volumetric resolution of fluorescence array tomography compares very favorably with the best optical sectioning microscopy methods. For a confocal microscope, the $z$-axis resolution is limited by diffraction to $\sim 700 \mathrm{~nm}$. The confocal's limiting $z$-axis resolution is usually worsened, however, by spherical aberration when a high-numerical-aperture (high-NA) objective is focused more deeply than a few micrometers into any tissue specimen. In contrast, the axial resolution limit for array tomography is simply the physical section thickness (typically $70 \mathrm{~nm}$ ). Physical sectioning for array tomography thus improves on ideal confocal optical sectioning by at least an order of magnitude. Spherical aberrations also adversely impact the lateral resolution of confocal microscopes because they are focused into a tissue depth: At focus depths of just a few micrometers, the degradation of lateral resolution can easily exceed a factor of 2 . Array tomography avoids this problem because the high-NA objective is always used at its design condition (i.e., immediate contact between the specimen and coverslip). Thus, array tomography using ordinary high-NA, diffraction-limited optics could conservatively improve volumetric resolution (i.e., the product of improvements in the $x-, y-$, and $z$-axes) by a factor of $40(2 \times 2 \times 10)$. Indeed, individual synapses in situ in the mammalian cortex generally cannot be resolved optically from their nearest neighbors by confocal microscopy but can be resolved quite reliably by array tomography (Micheva and Smith 2007).

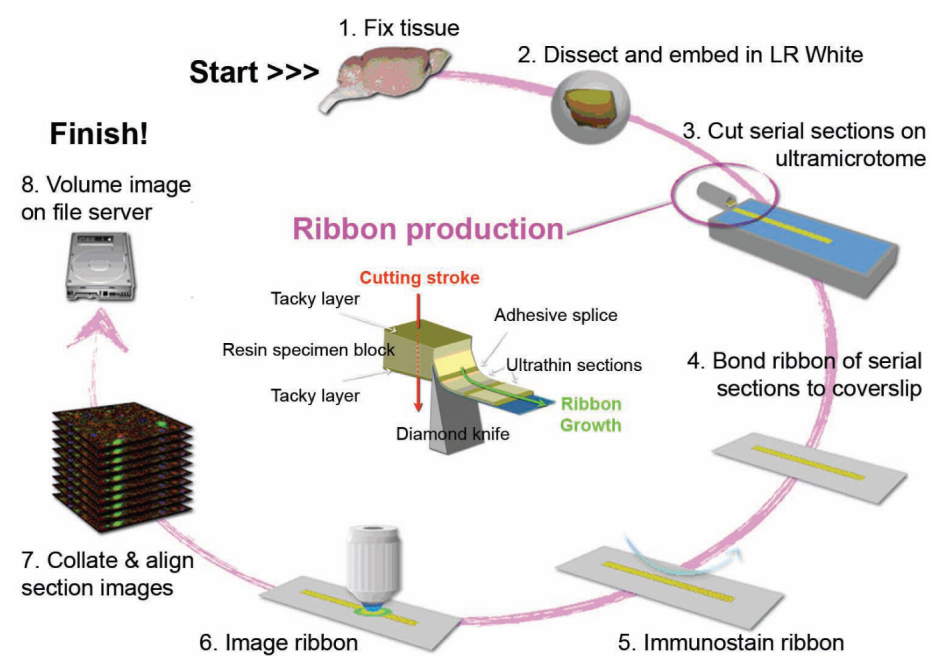

FIGURE 1. Sequence of steps for basic immunofluorescence array tomography. 


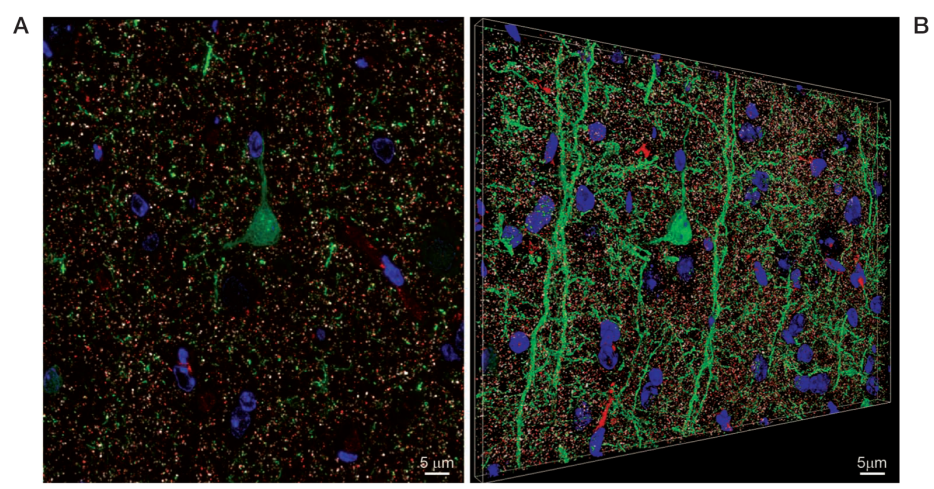

FIGURE 2. Array tomographic images of layer $\mathrm{V}$ neuropil in the barrel cortex of thy 1-YFP transgenic mouse (Feng et al. 2000). Yellow fluorescent protein is expressed in a subset of pyramidal cells (green). Synapsin 1 immunostaining and postsynaptic density protein 95 (PSD95) immunostaining are shown in white and red, respectively; 4',6-diamidine-2'phenylindole (DAPI) stains nuclear DNA blue. (A) Four-color fluorescence image of a single, ultrathin (200-nm) section. (B) Volume rendering of a stack of 30 sections after computational alignment. (For color figure, see doi: 10.1101/ pdb.top89 online at www.cshprotocols.org.)

\section{Depth Invariance}

The major limitation to quantitative interpretation of whole-mount tissue immunofluorescence images arises from reductions in both immunostaining and imaging efficiencies as focal plane depth increases. Diffusion and binding regimes typically limit the penetration of labeling antibodies to the first few micrometers below the surface of a tissue, even after prolonged incubations. Imaging efficiency likewise decreases with depth, because increasing spherical aberration and light scattering reduce signals profoundly with focal plane depths of just a few micrometers. These staining and imaging efficiency gradients make quantitative comparisons of specimen features at different depths with whole-mount (e.g., confocal) volume microscopy difficult and unreliable. Array tomography completely circumvents depth dependence issues, because each specimen volume element is stained identically as a result of minimal section thickness, and imaged identically because every section is bonded directly to the coverslip surface.

Multiplexity

Traditional multicolor immunofluorescence techniques have provided compelling evidence that multiple molecular species are localized at individual subcellular complexes. Because there are numerous (and diverse) distinct molecules at individual synapses, there is a pressing need for imaging techniques that can distinguish simultaneously more than the three or four species discernable using standard multicolor immunofluorescence. Attempts have been made to improve the multiplexity of immunofluorescence microscopy by repeated cycles of staining, imaging, and stain elution, but the results have been disappointing because the antibody elution treatments tend to destroy the samples. In array tomography, specimens are stabilized by the embedding resin matrix and by tight attachment to the coverslip substrate. An example of multiplexed staining with array tomography is shown in Figure 3, and thus far we have performed as many as nine cycles of staining, imaging, and elution (Micheva and Smith 2007). With four fluorescence "colors" per cycle, 36 or more antigens could be probed in one specimen. We now routinely acquire four colors in each of three cycles for a total of 12 marker channels. Although 12-36 markers might still fall short of the degree of multiplexing needed to fully probe the diverse molecules composing a synapse, this represents a substantial advance relative to traditional multicolor immunofluorescence methods.

\section{Volume Field of View}

In principle, array tomography offers unique potential for the acquisition of high-resolution volume images that extend "seamlessly" over very large tissue volumes. The depth invariance of array tomography noted above eliminates any fundamental limit to imaging in depth, whereas the availability of excellent automated image mosaic acquisition, alignment, and stitching algorithms allows tiling over arbitrarily large array areas. Ultimately, limits to the continuous arrayable volume can be imposed 

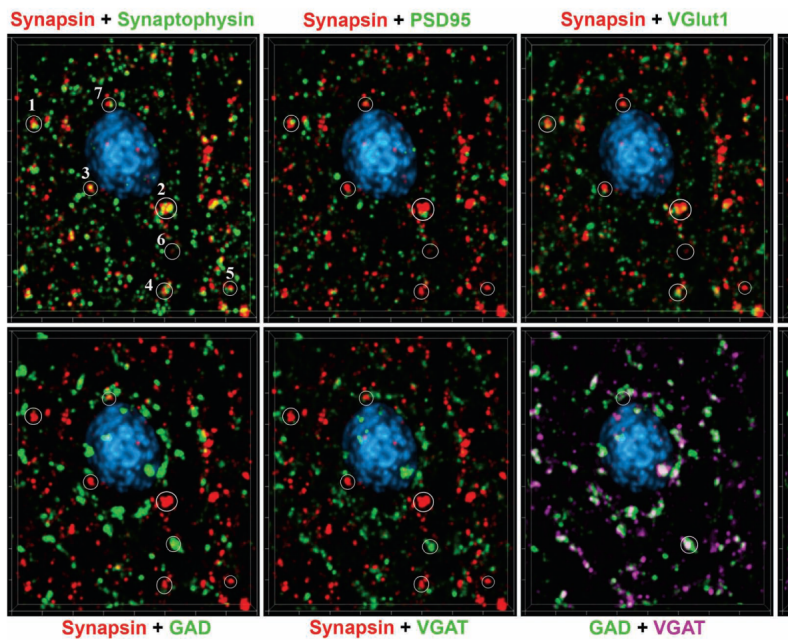

Synapsin + VGlut2

FIGURE 3. Multiplexed staining for seven synaptic proteins in mouse cerebral cortex (barrel cortex, layer 2/3) using five cycles of staining and elution. This volume $(18 \times 16 \times 1.3 \mu \mathrm{m})$ was reconstructed from 19 serial sections, $70 \mathrm{~nm}$ each. Individual synapsin puncta 1, 2, and 3 colocalize with synaptophysin and vesicular glutamate transporter 1 (VGlut1) and are closely apposed to PSD95 and thus appear to be excitatory synapses. Synapsin puncta 4-7 colocalize with synaptophysin, but do not have adjacent PSD95 puncta. Puncta 6 and 7 also colocalize with glutamic acid decarboxylase (GAD) and vesicular $\gamma$-aminobutyric acid transporter (VGAT) and thus have the characteristics of inhibitory synapses. (For color figure, see doi: 10.1101/pdb.top89 online at www.cshprotocols.org.)

by difficulties in tissue fixation, processing, and embedding (owing to diffusion limitations) as thicker volumes are encountered, and by mechanical issues (e.g., ultramicrotome and diamond knife engineering) as block face dimensions increase. Nevertheless, array tomography has already been performed successfully for volumes with minimum dimensions in the millimeter range, and it seems likely that volumes with minimum dimensions of several millimeters (e.g., an entire mouse brain) could eventually be manageable.

In practice, the size of seamless array tomography volumes is limited by the requirement that numerous steps in the fabrication, staining, and imaging of arrays be performed through many iterations without failure. At present, the most error-prone steps are those involved in array fabrication, whereas the most time-consuming are those involved in image acquisition. Ongoing engineering of array fabrication materials and processes will expand present limits to the error-free production of large arrays, whereas image acquisition times will be readily reducible by dividing large arrays across multiple substrates and imaging those subarrays on multiple microscopes.

\section{CONCLUSION AND FUTURE DIRECTIONS}

One important application of array tomography in the field of neuroscience is the analysis of synapse populations. With this method it is possible to resolve individual synapses in situ within brain tissue specimens. Because 10 or more antibodies can be used on an individual sample, the molecular signature of each synapse can be defined with unprecedented detail. The throughput of the technique is inherently high, approaching the imaging of one million synapses per hour. Compared with threedimensional reconstruction at the electron microscopic level, array tomography can image much larger volumes and provide information about the presence of a much larger number of molecules, but cannot presently provide the fine ultrastructural analysis of electron microscopy. On the other hand, the amount of effort involved in array tomography might not be warranted for all studies. If it is not considered critical to resolve individual synapses, immunostaining of vibratome sections or cryosections and confocal microscopy imaging may be sufficient.

Current work to enhance the utility of array tomography involves: (1) refining current staining and imaging approaches to image larger tissue volumes with more antibodies, (2) combining light and electron microscopic imaging to visualize both immunofluorescence and ultrastructure on the same tissue sections, and (3) applying advanced computational methods for data analysis, particularly to both count and classify millions of synapses on a routine basis. 


\section{ACKNOWLEDGMENTS}

We thank JoAnn Buchanan and Nafisa Ghori for their help in refining the methods. This work was supported by grants from McKnight Endowment Fund for the Neurosciences, the National Institutes of Health (NS 063210), The Gatsby Charitable Foundation, and the Howard Hughes Medical Institute.

\section{REFERENCES}

Feng G, Mellor RH, Bernstein M, Keller-Peck C, Nguyen QT, Wallace M, Nerbonne JM, Lichtman JW, Sanes JR. 2000. Imaging neuronal subsets in transgenic mice expressing multiple spectral variants of GFP. Neuron 28: 41-51.

Koffie RM, Meyer-Luehmann M, Hashimoto T, Adams KW, Mielke ML, Garcia-Alloza M, Micheva KD, Smith SJ, Kim ML, Lee VM, et al. 2009. Oligomeric amyloid $\beta$ associates with postsynaptic densities and correlates with excitatory synapse loss near senile plaques. Proc Natl Acad Sci 106: 4012-4017.

Micheva KD, Smith SJ. 2007. Array tomography: A new tool for imaging the molecular architecture and ultrastructure of neural circuits. Neuron 55: 25-36.

Micheva KD, O'Rourke N, Busse B, Smith SJ. 2010a. Array tomography: Rodent brain fixation and embedding. Cold Spring Harb Protoc doi: 10.1101/pdb.prot5523.

Micheva KD, O'Rourke N, Busse B, Smith SJ. 2010b. Array tomography: Production of arrays. Cold Spring Harb Protoc doi: 10.1101/ pdb.prot5524.

Micheva KD, O'Rourke N, Busse B, Smith SJ. 2010c. Array tomography: Immunostaining and antibody elution. Cold Spring Harb Protoc doi: 10.1101/pdb.prot5525.

Micheva KD, O'Rourke N, Busse B, Smith SJ. 2010d. Array tomography: Imaging stained arrays. Cold Spring Harb Protoc doi: $10.1101 /$ pdb.prot5526.

Micheva KD, O’Rourke N, Busse B, Smith SJ. 2010e. Array tomography: Semiautomated image alignment. Cold Spring Harb Protoc doi: $10.1101 /$ pdb.prot5527.

Smith SJ. 2007. Circuit reconstruction tools today. Curr Opin Neurobiol 17: 601-608.

Stevens B, Allen NJ, Vazquez LE, Howell GR, Christopherson KS, Nouri N, Micheva KD, Mehalow A, Huberman AD, Stafford B, et al. 2007. The classical complement cascade mediates CNS synapse elimination. Cell 131: 1164-1178. 


\section{Array Tomography: High-Resolution Three-Dimensional Immunofluorescence}

Kristina D. Micheva, Nancy O'Rourke, Brad Busse and Stephen J. Smith

Cold Spring Harb Protoc; doi: 10.1101/pdb.top89

\begin{tabular}{|c|c|}
\hline $\begin{array}{r}\text { Email Alerting } \\
\text { Service }\end{array}$ & Receive free email alerts when new articles cite this article - click here. \\
\hline $\begin{array}{l}\text { Subject } \\
\text { Categories }\end{array}$ & $\begin{array}{l}\text { Browse articles on similar topics from Cold Spring Harbor Protocols. } \\
\text { Antibodies, general (289 articles) } \\
\text { Cell Biology, general (1382 articles) } \\
\text { Cell Imaging (525 articles) } \\
\text { Computational Biology (100 articles) } \\
\text { Fluorescence (517 articles) } \\
\text { Fluorescence, general (341 articles) } \\
\text { Fluorescent Proteins (259 articles) } \\
\text { High-Throughput Analysis, general (155 articles) } \\
\text { Image Analysis (124 articles) } \\
\text { Imaging Development ( } 255 \text { articles) } \\
\text { Imaging for Neuroscience (342 articles) } \\
\text { Imaging/Microscopy, general (579 articles) } \\
\text { Immunohistochemistry (92 articles) } \\
\text { Immunoimaging (41 articles) } \\
\text { Immunostaining (139 articles) } \\
\text { Immunostaining, general (94 articles) } \\
\text { Proteins and Proteomics, general (575 articles) } \\
\text { Visualization (524 articles) } \\
\text { Visualization of Proteins (107 articles) } \\
\text { Visualization, general (369 articles) }\end{array}$ \\
\hline
\end{tabular}

